\title{
Surgical treatment for oral tongue squamous cell carcinoma: A retrospective study of 432 patients
}

\author{
Yu Oikawa*, Takuma Kugimoto, Yoshihisa Kashima, Kohei Okuyama, Toshimitsu Ohsako, Takeshi Kuroshima, \\ Hideaki Hirai, Hirofumi Tomioka, Hiroaki Shimamoto, Yasuyuki Michi, Hiroyuki Harada
}

Department of Oral and Maxillofacial Surgery, Graduate School of Medical and Dental Sciences, Tokyo Medical and Dental University, Tokyo, Japan.

\begin{abstract}
The incidence of oral cancer in Japan accounts for $1 \%$ of all cancers, with oral tongue cancer accounting for $60 \%$ of oral cancers based on the subsite. The most common histologic type is squamous cell carcinoma. This study aimed to evaluate the series of surgical treatments for 432 patients with oral tongue squamous cell carcinoma (OTSCC). Initial surgical treatments for the primary site included partial glossectomy, hemiglossectomy, and total or subtotal glossectomy in 348, 58, and 26 patients, respectively. Therapeutic neck dissection, elective neck dissection, and subsequent neck dissection were performed in 74, 53, and 37 patients, respectively. Patients with advanced cases had level IIb, IV, and V metastasis and outside regional lymph node metastases. The cumulative 5-year disease-specific survival rate for OTSCC was $92.8 \%$, and the rates for each stage were $96.6 \%, 93.9 \%, 84.1 \%$, and $79.0 \%$ in stages I, II, III, and IV, respectively. The recurrence rate, overall salvage rate for recurrent cases, and rate for the additional surgical group were 10.4\%, 46.7\%, and 78.6\%, respectively. Patients with multiple cervical lymph node metastases, extranodal extension, metastases to multiple levels, and lower neck metastases had poor prognosis. In conclusion, careful follow-up is necessary to detect recurrence of primary tumors at a stage when surgical treatment can be performed, and cervical lymph node status is one of the most important prognostic factors in OTSCC.
\end{abstract}

Keywords: oral cancer, oral tongue squamous cell carcinoma (OTSCC), surgical treatment, neck dissection

\section{Introduction}

Annually, approximately 300,000 people worldwide develop oral cancer (1). The majority of oral malignancies arise from epithelial tissue, and squamous cell carcinoma is the predominant tumor type (2). The most common subsite of oral squamous cell carcinoma (OSCC) in Japan is the oral tongue, and the estimated frequency of oral tongue squamous cell carcinoma (OTSCC) is $60 \%$ (3). Although the oral tongue can be inspected directly, it is often diagnosed in its advanced stages. The oral tongue controls functions such as mastication, taste, articulation, and deglutition; hence, the treatment of OTSCC has a great impact on the postoperative dysfunction as well as life prognosis. Despite advances in diagnostics and therapeutic techniques, the survival rate of OSCC has only improved by $5 \%$ in the past 20 years, and the 5 -year survival rate of OSCC is $60 \%$ (4).

Few reports have examined the course of treatment and prognosis in patients with OTSCC. This study aimed to evaluate the series of treatments for OTSCC and their outcomes in a single institution.

\section{Patients and Methods}

Between January 2008 and December 2017, 778 patients with OSCC underwent radical surgical treatment at the Department of Oral and Maxillofacial Surgery of the Tokyo Medical and Dental University (Tokyo, Japan). Among these patients, 432 (55.5\%) had OTSCC. The observation period was set from the date of treatment initiation to December 31, 2019, and the mean followup period was 70.5 months (range, 1.6-141.4 months). Patients with multiple oral cancers and treated with brachytherapy were excluded. Clinicopathological information including age, sex, TNM classification (Union for International Cancer Control 7th edition), treatment, and outcomes were obtained from medical charts. Speech intelligibility was assessed using a hundred monosyllable Japanese speech intelligibility test (5), and each patient read all items on the list twice. Five independent listeners scored speech intelligibility, and the mean scores of three of these listeners (excluding the highest and lowest scores) were used as a measure of speech intelligibility.

Survival curves were estimated according to the 
Kaplan-Meier method, and differences were examined using the log-rank test. The analyses were performed using PASW Statistics version 25 (SPSS Inc., Chicago, IL, USA).

This study was conducted following the Declaration of Helsinki and was approved by the ethics committee of the Tokyo Medical and Dental University, Faculty of Dentistry (No. D2015-600).

\section{Results}

\section{Initial treatment for primary site and neck}

There were 264 males and 168 females among the 432 patients, and the mean age was 57.8 years (range, 21-86 years). Among both males and females, the highest number of patients were in their 60 s (65 and 38 patients, respectively). Tumor clinical stages were stage I in 235 patients, stage II in 108 patients, stage III in 40

\section{Table 1. Characteristics of patients}

\begin{tabular}{lc}
\hline Variables & Patients \\
\hline Sex & \\
Male & 264 \\
Female & 168 \\
Age years (mean) & $21-86(57.8)$ \\
Stage & \\
I & 235 \\
II & 108 \\
III & 40 \\
IVA & 49 \\
Treatment for primary & \\
Surgical only & 360 \\
Adjuvant therapy & 36 \\
Neoadjuvant therapy & 17 \\
Adjuvant and neoadjuvant therapy & 19 \\
Neck dissection & \\
Therapeutic neck dissection & 74 \\
Elective neck dissection & 53 \\
Subsequent neck dissection & 37 \\
Type of recurrence & 17 \\
Local recurrence & 22 \\
Regional recurrence & 6 \\
Locoregional recurrence & \\
\hline
\end{tabular}

patients, and stage IVA in 49 patients (Table 1).

The initial treatment for the primary cancer consisted of surgery alone in 360 patients, adjuvant therapy in 36 patients, neoadjuvant therapy in 17 patients, and adjuvant and neoadjuvant therapy in 19 patients (Table 1). Neoadjuvant therapy consisted of chemotherapy in 16 patients and chemoradiotherapy in 20 patients. As for adjuvant therapy, 11 patients received chemotherapy, 14 patients received radiotherapy, and 30 patients received chemoradiotherapy.

Initial surgical treatment for the primary site consisted of partial glossectomy in 348 patients, hemiglossectomy in 58 patients, and total or subtotal glossectomy in 26 patients. We performed combined adjacent tissue resection for 18 patients in the hemiglossectomy group (combined marginal mandibulectomy in 5 patients, lateral pharyngectomy in 11 patients, and both in 2 patients) and 14 patients in the total or subtotal glossectomy group (combined marginal mandibulectomy in 6 patients, lateral pharyngectomy in 4 patients, and both in 4 patients). Table 2 shows the reconstruction type of the primary site. Primary closure was the most common type in the partial glossectomy group $(82.8 \%)$, radial forearm flap in the hemiglossectomy group (84.5\%), and rectus abdominis myocutaneous flap in the total or subtotal glossectomy group (65.4\%).

Six patients had histopathologically positive margins on the surgical specimen of the primary tumor, 5 patients underwent additional resections, and 1 patient received chemoradiotherapy. None of the patients had local recurrence.

Neck dissection was performed in 127 patients as the initial treatment. In these patients, 74 underwent therapeutic neck dissection (62 unilateral, 12 bilateral) and 53 underwent elective neck dissection (Table 1). Among patients who underwent therapeutic neck dissection, $61(82.4 \%)$ had cervical lymph node metastases identified pathologically; in contrast, among those who underwent elective neck dissection, 13 patients $(24.5 \%)$ had cervical lymph node metastases. Thirtynine patients, excluding 2 patients who had primary recurrence, developed subsequent cervical lymph node

Table 2. Initial treatment of primary site and reconstruction

\begin{tabular}{lrrrrrr}
\hline Variables & $\begin{array}{c}\text { Partial glossectomy } \\
(n=348)\end{array}$ & \multicolumn{2}{c}{$\begin{array}{c}\text { Hemiglossectomy } \\
(n=58)\end{array}$} & \multicolumn{2}{c}{$\begin{array}{c}\text { Total or subtotal glossectomy } \\
(n=26)\end{array}$} \\
\hline Primary closure & 288 & $(82.8 \%)$ & - & $(0 \%)$ & - & $(0 \%)$ \\
STSG & 15 & $(4.3 \%)$ & 1 & $(1.7 \%)$ & - & $(0 \%)$ \\
PGA sheet & 16 & $(4.6 \%)$ & 1 & $(1.7 \%)$ & - & $(0 \%)$ \\
Artificial dermi & 2 & $(0.6 \%)$ & - & $(0 \%)$ & - & $(0 \%)$ \\
RF flap & 27 & $(7.8 \%)$ & 49 & $(84.5 \%)$ & 4 & $(15.4 \%)$ \\
ALT flap & - & $(0 \%)$ & 2 & $(3.4 \%)$ & 2 & $(7.7 \%)$ \\
TFL flap & - & $(0 \%)$ & 1 & $(1.7 \%)$ & 3 & $(11.5 \%)$ \\
RA flap & - & $(0 \%)$ & 3 & $(5.2 \%)$ & 17 & $(65.4 \%)$ \\
LDMC and scapular flap & - & $(0 \%)$ & 1 & $(1.7 \%)$ & - & $(0 \%)$ \\
\hline
\end{tabular}

ALT: anterolateral thigh; LDMC: lattissimus dorsi musculocutaneous; PGA: polyglycolic acid; RA: rectus abdominis myocutaneous; RF: radial forearm; STSG: split thickess skin graft; TFL: tensor fasciae latae. 
Table 3. Level of metastatic lymph nodes $(n=111)$

\begin{tabular}{lrrrr}
\hline Level & \multicolumn{2}{c}{ Ipsilateral } & \multicolumn{2}{l}{ Contralateral } \\
\hline Ia & 16 & $(14.4 \%)$ & 3 & $(2.7 \%)$ \\
Ib & 46 & $(41.4 \%)$ & 8 & $(7.2 \%)$ \\
IIa & 79 & $(71.2 \%)$ & 3 & $(2.7 \%)$ \\
IIb & 4 & $(3.6 \%)$ & - & $(0 \%)$ \\
III & 39 & $(35.1 \%)$ & 4 & $(3.6 \%)$ \\
IV & 9 & $(8.1 \%)$ & 1 & $(0.9 \%)$ \\
V & 3 & $(2.7 \%)$ & - & $(0 \%)$ \\
Others & 15 & $(13.5 \%)$ & - & $(0 \%)$ \\
\hline
\end{tabular}

metastases. The mean duration of metastases, which appeared clinically was 8.5 months (range, 0.7-32.9 months). In these patients, 37 underwent therapeutic neck dissection and the other 2 were treated in other institutions. Among the 37 patients, 12 were treated with adjuvant therapy.

Among the 164 patients who underwent neck dissection, 111 had histopathological lymph node metastases. Of these, 94 patients had unilateral neck dissection and 17 patients had bilateral neck dissection. The patterns of cervical lymph node metastases are shown in Table 3. In the ipsilateral site, level IIa was the most common $(71.2 \%)$, while level $\mathrm{Ib}$ was the most common in the contralateral site $(7.2 \%)$. There was a low frequency of metastases to the ipsilateral levels IIb $(3.6 \%)$, IV $(8.1 \%)$, and V $(2.7 \%)$, and were found in only 13 patients. The mean number of metastatic lymph nodes in patients with levels IIb, IV, and V regional metastases was 10.5 (range, 3-28), and 5 out of 13 patients developed distant metastases. Skip metastasis to level III, without involvement of levels I or II, was confirmed in 9 patients $(8.1 \%)$. Metastases outside the regional lymph nodes were observed in 15 patients. Thirteen patients had metastasis to the lingual nodes, while there was one patient each who had metastasis to the intraglandular parotid nodes and paratracheal nodes. Lastly, 8 out of 15 patients developed distant metastases.

\section{Recurrence and salvage treatment}

Seventeen patients had local recurrences, 22 had regional recurrences, and 6 had locoregional recurrences. The recurrence rate of OTSCC was $10.4 \%$. In local recurrences, 11 patients underwent additional surgical treatment and 10 patients were salvaged. Conversely, in the 6 patients who received chemotherapy and/or radiotherapy, only 2 survived. In regional recurrence, 17 patients had recurrence beyond the dissected field. Sixteen patients underwent surgery, of which 11 were salvageable. Six patients who received chemotherapy and/or radiotherapy had unfavorable outcomes. In locoregional recurrence, only one patient could be salvaged with neck surgery and proton radiation therapy to the primary recurrent tumor. The overall salvage rate for recurrent cases was $46.7 \%$, and the rate for the additional surgical group was $78.6 \%$. Distant metastases were found in 19 patients $(4.4 \%), 9$ of which gained locoregional control.

\section{Postoperative function}

The speech intelligibility test was performed in 296 patients $(68.5 \%)$, and the mean period from surgery to speech evaluation was 21.6 days (range, 3-143 days). The mean scores were $92.9 \%$ (range, $41.0-100 \%$ ) in the partial glossectomy group $(n=216), 79.1 \%$ (range, $32.7-97.3 \%)$ in the hemiglossectomy group $(n=55)$, and $49.0 \%$ (range, 24.3-83.3\%) in the total or subtotal glossectomy group $(n=25)$.

The mean time from initial surgery to oral food intake was 6.0 days (range, 0-27 days) in the partial glossectomy group, 22.0 days (range, 6-92 days) in the hemiglossectomy group, and 29.2 days (range, 14127 days) in the total or subtotal glossectomy group, excluding 4 patients who were fed via percutaneous endoscopic gastrostomy (PEG). The four patients who underwent PEG consisted of 2 patients with hemiglossectomy, 1 patient with subtotal glossectomy, and 1 patient with total glossectomy.

\section{Outcomes of surgical treatment of OTSCC}

The cumulative 5-year overall survival and diseasespecific survival (DSS) rates for OTSCC were 91.1\% and $92.8 \%$, respectively. The rate of DSS for each stage was $96.6 \%$ in stage I, $93.9 \%$ in stage II, $84.1 \%$ in stage III, and $79.0 \%$ in stage IV (Figure 1). The rate of DSS with cervical lymph node metastases was $76.6 \%$ ( $n=$ $111)$ and $98.2 \%$ in negative lymph nodes metastasis $(n=321)(p<0.01)$ (Figure 2). DSS rates were also calculated for the metastatic pattern of the cervical lymph nodes. The rates for single and multiple lymph node metastases were $89.9 \%(n=47)$ and $66.9 \%(n=$ 64) $(p=0.013)$, with and without extranodal extension were $68.1 \%(n=59)$ and $85.2 \%(n=52)(p=0.011)$, single and multiple levels of metastases were $89.1 \%$

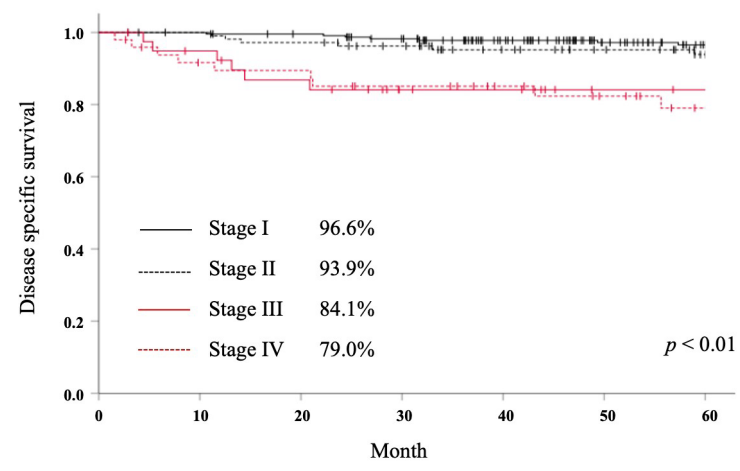

Figure 1. Kaplan-Meier estimates of disease-specific survival (DSS) among patients with oral tongue squamous cell carcinoma (OTSCC) according to the tumor clinical stage. 
$(n=62)$ and $60.3 \%(n=49)(p=0.002)$, and with and without lower neck involvement (level IV and/or V) were $42.4 \%(n=11)$ and $80.4 \%(n=100)(p=0.005)$, respectively (Figure 3 ).

\section{Discussion}

The morbidity of oral cancers and the frequency of occurrence by subsite vary by country, race, and lifestyle. The incidence of oral cancer in Japan is reported to be $1 \%$ of all cancers, and the number of patients is increasing with the age of the population (4). In Western countries, the number of patients with oral cancer among young people and nonsmokers is on the rise (6), but no such trend has been observed in Japan.

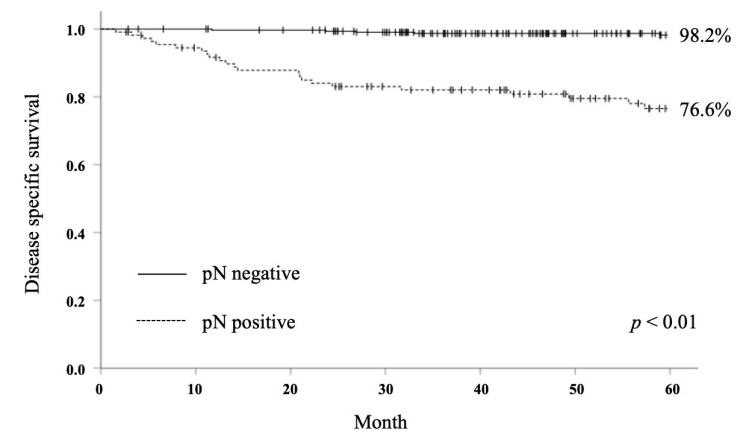

Figure 2. Kaplan-Meier estimates of disease-specific survival (DSS) among patients according to the presence or absence of the cervical lymph node metastasis.

A

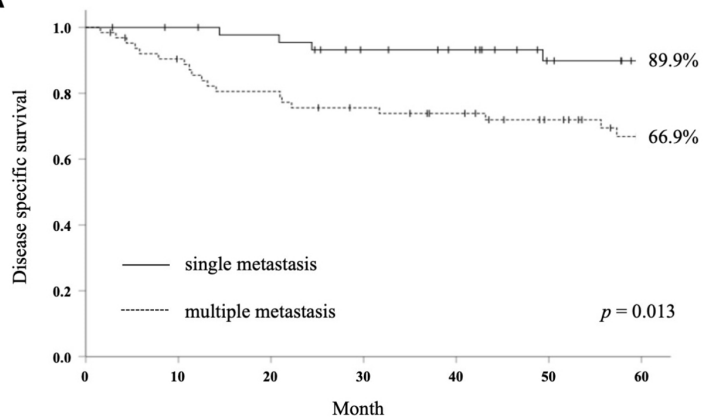

C

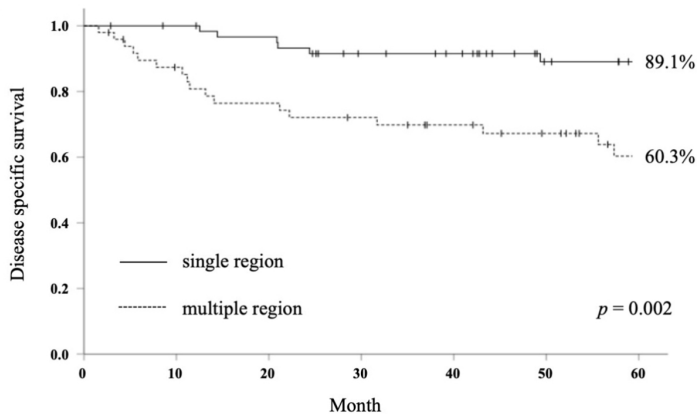

Surgical resection of the oral tongue impairs its primary functions such as articulation, mastication, and swallowing. In partial glossectomy or hemiglossectomy resection cases, it is desirable to reconstruct the tongue without impairing the function of the remaining tongue. In total or subtotal resection cases, on the other hand, it is desirable to reduce the space between the reconstructed tongue and the palate to restore articulation and swallowing functions (3). For this reason, the radial forearm flap is often used in patients with hemiglossectomy of the tongue (7) and the rectus abdominis myocutaneous flap is used in patients with total or subtotal resection of the tongue (8). Our strategy of reconstruction according to the extent of resection was similar to these results.

As an assessment of function after tongue resection and reconstruction, we tested for articulatory function. It is clear from the present study that as the extent of resection increases, articulatory function declines. Similarly, the time until initiation of oral food intake varies with the extent of resection. At our facility, the swallowing function is assessed postoperatively by video fluoroscopy, and indirect training, direct training, and use of palatal augmentation prosthesis are performed as needed. This allows most patients to eat through the oral route before they leave the hospital. In addition to the type of flap, the design of the flap and the method should be considered to achieve good postoperative oral function. In reconstruction of a large excision of the tongue, a protuberant shape is useful
B

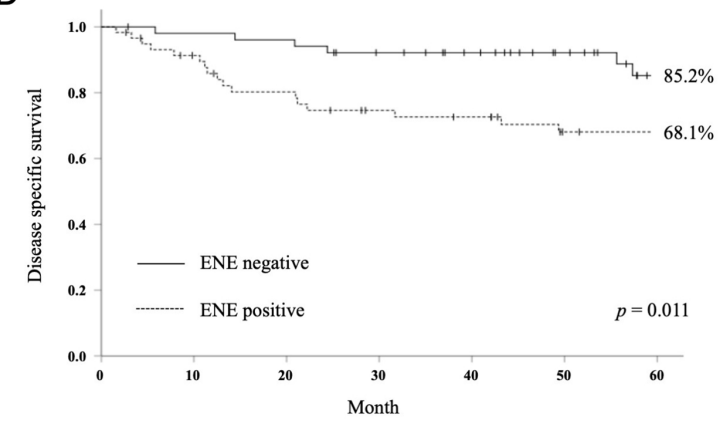

D

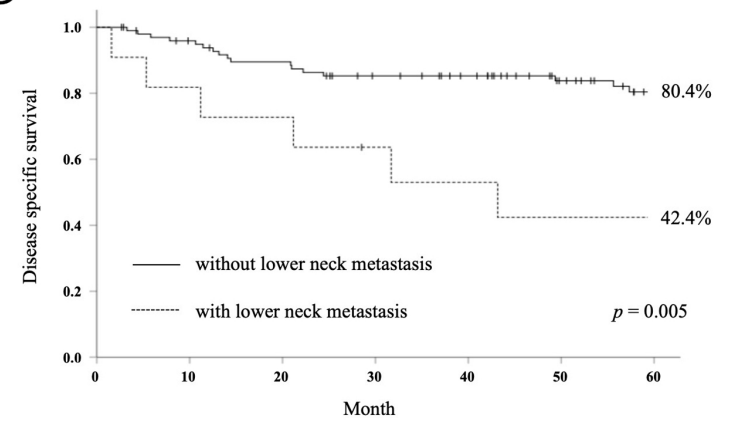

Figure 3. Kaplan-Meier estimates of disease-specific survival (DSS) among patients according to the metastatic pattern of the cervical lymph nodes. (A) Single and multiple lymph node metastasis. (B) With and without extranodal extension. (C) Single and multiple levels of metastasis. (D) With and without lower neck (level IV and/or V) metastasis. 
in preserving swallowing and articulation function after surgery $(9,10)$, and laryngeal suspension is also performed to prevent subsidence of the reconstructed tongue (11).

There is a lack of consensus regarding elective neck dissection. Although the metastasis rate of patients undergoing elective neck dissection in this study was $24.5 \%$, the metastatic rate in early stage cancers is unknown because our institution performs elective neck dissection in cases of locally advanced cancer that require free flap reconstruction. Even after elective neck dissection, cervical recurrence can occur (12), and further studies on the indications for elective neck dissection, the extent of dissection, postoperative treatment, and follow-up are needed. Cervical lymph node metastasis in oral cancer was more common in levels I and II (13), which was also the case in the present study, metastasis to regions with low frequency of lymph node metastasis, such as level IIb, IV, and V, tended to show distant metastases. Risk factors involved in neck failure include multiple cervical lymph node metastases, extranodal extension, metastases to multiple levels, and lower neck metastases $(14,15)$. In the present study, significant differences were found for all factors; however, the analysis of poor prognostic factors in this study included both patients who received adjuvant therapy and those who did not. Adjuvant therapy is often administered to patients who have poor prognostic factors, and it is assumed that there are some factors that cannot be controlled even with adjuvant therapy; hence, future research should focus on resistance to adjuvant therapy.

Surgery is generally the treatment of choice for recurrent cancer because it offers a better prognosis than other therapies if the cancer is resectable (16). We surgically treated 28 out of 45 patients with recurrence and 22 had favorable outcomes. In contrast, the prognosis for unresectable recurrent tumors is poor, with a low survival rate. It is more important to detect recurrence when surgical treatment is possible. At our institution, CT or PET/CT are routinely performed once or twice a year for at least 2 years after primary treatment or neck dissection.

In recent years, comprehensive analyses of gene mutations in head and neck squamous cell carcinoma (HNSCC) including OSCC have also been conducted, revealing substantial information about the genomic alterations in HNSCC (17). We also reported that receptor tyrosine kinase amplification or co-expression of EGFR and MET was associated with poor prognosis $(18,19)$. However, these findings have not yet been translated into clinical applications. Further research is needed to explore biomarkers for treatment tolerance and prognosis prediction, and their clinical applications to implement precise medical care by stratifying patients in oral cancer.

In conclusion, careful follow-up is necessary to detect recurrent primary tumors at a stage when surgical treatment can still be performed. Tumor cells in OTSCC often metastasize to cervical lymph node levels Ia and IIa. However, metastasis to level IIb and the lower cervical region was also found in patients with multiple metastases, and occult metastases were found in locally advanced cases. Cervical lymph node status is one of the most important prognostic factors in OTSCC. Finally, further studies regarding treatment strategies are needed in the future.

\section{Funding: None.}

Conflict of Interest: The authors have no conflicts of interest to disclose.

\section{References}

1. Ferlay J, Soerjomataram I, Dikshit R, Eser S, Mathers C, Rebelo M, Parkin DM, Forman D, Bray F. Cancer incidence and mortality worldwide: sources, methods and major patterns in GLOBOCAN 2012. Int J Cancer. 2015;136:E359-386.

2. World Health Organization Classification of Tumours. Pathology and Genetics of Head and Neck Tumors (Barnes L, Eveson JW, Reichart P, Sidransky D, eds.). IARC Press. Lyon, France, 2005.

3. Ariyoshi Y, Shimahara M, Omura K, Yamamoto E, Mizuki H, Chiba H, Imai Y, Fujita S, Shinohara M, Seto K; Japanese Society of Oral and Maxillofacial Surgeons, 2002. Epidemiological study of malignant tumors in the oral and maxillofacial region: survey of member institutions of the Japanese Society of Oral and Maxillofacial Surgeons, 2002. Int J Clin Oncol. 2008; 13:220-228.

4. Chinn SB, Myers JN. Oral cavity carcinoma: current management, controversies, and future directions. J Clin Oncol. 2015; 33:3269-3276.

5. Furuya G. Study on the articulation test in pronunciation in speech disorders. Nihon Jibiinkoka Gakkai Kaiho. 1958; 61:1922-1948.

6. Patel SC, Carpenter WR, Tyree S, Couch ME, Weissler M, Hackman T, Hayes DN, Shores C, Chera BS. Increasing incidence of oral tongue squamous cell carcinoma in young white women, age 18 to 44 years. J Clin Oncol. 2011; 29:1488-1494.

7. Hsiao HT, Leu YS, Lin CC. Primary closure versus radial forearm flap reconstruction after hemiglossectomy: functional assessment of swallowing and speech. Ann Plast Surg. 2002; 49:612-616.

8. Lyos AT, Evans GR, Perez D, Schusterman MA. Tongue reconstruction: outcomes with the rectus abdominis flap. Plast Reconstr Surg. 1999; 103:442-447; discussion 448449.

9. Kimata Y, Sakuraba M, Hishinuma S, Ebihara S, Hayashi R, Asakage T, Nakatsuka T, Harii K. Analysis of the relations between the shape of the reconstructed tongue and postoperative functions after subtotal or total glossectomy. Laryngoscope. 2003; 113:905-909.

10. Kiyokawa K, Tai Y, Inoue Y, Yanaga H, Mori K, Nakashima T. Functional reconstruction of swallowing and articulation after total glossectomy without 
laryngectomy: money pouch-like reconstruction method using rectus abdominis myocutaneous flap. Plast Reconstr Surg. 1999; 104:2015-2020.

11. Goode RL. Laryngeal suspension in head and neck surgery. Laryngoscope. 1976; 86:349-355.

12. Shimamoto H, Oikawa Y, Osako T, Hirai H, Mochizuki Y, Tanaka K, Tomioka H, Harada H. Neck failure after elective neck dissection in patients with oral squamous cell carcinoma. Oral Surg Oral Med Oral Pathol Oral Radiol. 2017; 124:32-36.

13. Shah JP, Candela FC, Poddar AK. The patterns of cervical lymph node metastases from squamous carcinoma of the oral cavity. Cancer. 1990; 66:109-113.

14. Shingaki S, Nomura T, Takada M, Kobayashi T, Suzuki I, Nakajima T. The impact of extranodal spread of lymph node metastases in patients with oral cancer. Int J Oral Maxillofac Surg. 1999; 28:279-284.

15. Mamelle G, Pampurik J, Luboinski B, Lancar R, Lusinchi A, Bosq J. Lymph node prognostic factors in head and neck squamous cell carcinomas. Am J Surg. 1994; 168:494-498.

16. Schwartz GJ, Mehta RH, Wenig BL, Shaligram C, Portugal LG. Salvage treatment for recurrent squamous cell carcinoma of the oral cavity. Head Neck. 2000; 22:34-41.

17. Cancer Genome Atlas Network. Comprehensive genomic characterization of head and neck squamous cell carcinomas. Nature. 2015; 517:576-582.

18. Oikawa Y, Morita KI, Kayamori K, Tanimoto K, Sakamoto K, Katoh H, Ishikawa S, Inazawa J, Harada H. Receptor tyrosine kinase amplification is predictive of distant metastasis in patients with oral squamous cell carcinoma. Cancer Sci. 2017; 108:256-266.

19. Yokokawa M, Morita KI, Oikawa Y, Kayamori K, Sakamoto K, Ikeda T, Harada H. Co-expression of EGFR and MET has a synergistic effect on the prognosis of patients with oral squamous cell carcinoma. J Oral Pathol Med. 2020; 49:235-242.

Received September 28, 2020; Revised February 15, 2021; Accepted February 22, 2021.

Released online in J-STAGE as advance publication March 6, 2021.

*Address correspondence to:

Yu Oikawa, Department of Maxillofacial Surgery, Graduate School of Medical and Dental Sciences, Tokyo Medical and Dental University, 1-5-45 Yushima, Bunkyo, Tokyo 113-8510, Japan.

E-mail: oikawa.osur@tmd.ac.jp 